\title{
Electromyographical and Physiological Correlation in Patient with Heart Disease
}

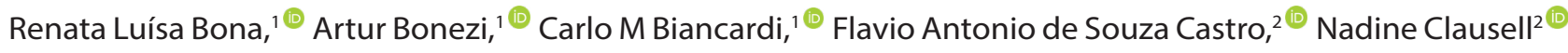 \\ Universidad de la Republica Uruguay - Centro Universitario de Paysandu, ${ }^{2}$ Paysandú - Uruguay \\ Universidade Federal do Rio Grande do Sul, ${ }^{2}$ Porto Alegre, RS - Brazil
}

\section{Abstract}

Background: Walking is an economic activity, the more efficient the mechanical contribution, the less metabolic energy is necessary to keep walking. Patients with chronic heart failure and heart transplant present peripheral musculoskeletal disorders, dyspnea, and fatigue in their activities.

Objective: In this scenario, the present study sought to verify the correlations between metabolic and electromyographic variables in chronic heart failure, heart transplant patients, and healthy controls.

Methods: Regression and correlation between cost of transport and electromyographic cost, as well as correlation between oxygen consumption and muscle coactivation in patients and controls at five different walking speeds have been performed, with alpha $=0.05$.

Results: Strong correlation values (r controls: 0.99; chronic heart failure: 0.92; heart transplant: 0.88) indicate a linear relationship between the cost of transport and electromyographic cost. Oxygen consumption was significantly correlated to muscle activation in all groups.

Conclusion: These results suggested that dynamic muscle coactivation was an important factor, especially for $\mathrm{CHF}$ and HT. These data support the idea that peripheral muscle limitations play an important role in people with $\mathrm{CHF}$ and HT. These findings indicate a strong relation between metabolic and electromyographic variables. For chronic heart failure and heart transplant patients, it can help to explain some difficulties in daily activities and aid in physical rehabilitation.

Keywords: Heart Failure; Heart Transplantation; Electromyographic/methods; Transportation; Costs and Analysis; Gait; Walking; Running.

\section{Introduction}

Walking and running are the most common human gaits. Humans walk at low speed and change gait to increase their locomotion speed, minimizing the energy expenditure. Thus, at speeds below walk-run transition speed, the oxygen consumption of walking is lower than that of running, while at higher speeds the relationship is reversed. ${ }^{1}$ The energetic cost to travel a given distance is called the cost of transport (C), and has long been known to strongly depend on speed in human walking. ${ }^{2,3}$ The cost is minimized at intermediate walking speeds of $4.5-5.4 \mathrm{~km} \cdot \mathrm{h}^{-1}$ (or $1.25-1.5 \mathrm{~m} \cdot \mathrm{s}^{-1}$ ) and grows as speed increases above or decreases below this optimum value. This trend is related to the pendular transfer of kinetic and potential energy, which is greater at intermediate speeds, ${ }^{4}$ reducing the total mechanical work that must be performed by muscles at these speeds. Equally, the described energetically optimal walking speeds are in accordance with the contractile physiology of skeletal muscle and the biomechanical models of terrestrial locomotion. ${ }^{5}$

Humans are exceptionally economical walkers 2,6 with their long legs and capacity to store and recover elastic strain energy. ${ }^{7}$ If the activity of individual muscles is minimized at intermediate walking speeds, as suggested above, we would expect the various 
muscles of the human locomotor system to be tuned to the same optimal speed to reduce the energetic cost of locomotion. ${ }^{5}$

Patients with chronic heart failure (CHF) and heart transplant (HT) experience fatigue and dyspnea during daily activities as well as during maximal exercise testing..$^{8,9} \mathrm{HT}$ is the preferred therapeutic strategy for the treatment of end-stage CHF. HT patients have different responses to physical exercise because of sustained pre-transplant physical conditions. Wilson el al., ${ }^{8}$ suggested that nutritive flow to skeletal muscles was defective in patients with $\mathrm{CHF}$, and that their diminished maximal exercise capacity was correlated to the degree of impairment of nutrient flow. The exertional fatigue suffered by these patients was attributed to skeletal muscle underperfusion. ${ }^{8,}{ }^{10}$ However, Wilson et al., ${ }^{11}$ demonstrated that patients with $\mathrm{CHF}$ and exertional fatigue were limited by skeletal muscle abnormalities rather than by skeletal muscle underperfusion. Further studies indicated that early skeletal muscle anaerobic metabolism, with lactate production, is the primary factor limiting exercise performance in patients with this disorder. ${ }^{9,11}$ The skeletal muscle metabolic response to exercise is altered in patients with CHF.

Studies with EMG activity in CHF patients demonstrated that surface EMG can be used to detect skeletal muscle fatigue, altered muscle activity and atrophy. ${ }^{12,13}$ Information of muscle activity in groups of $\mathrm{CHF}$ and HT showed high coactivation and high "global" EMG (called electromyographic cost) when compared to healthy controls. ${ }^{14}$ The correlation between metabolic and electromyographic variables can help to understand the changes in patients walking parameters and propose adequate goals during physical rehabilitation.

It is known that patients with cardiac diseases undergo musculoskeletal peripheral disorders, as well as anomalies in oxygen consumption, cost of transport, electromiographic activity and muscular coactivation. ${ }^{11,}$

${ }^{13}$ In a previous study, it has been shown that ventilatory efficiency disorders in CHF patients could explain some changes during physical activity. ${ }^{15}$ However, others factors should be taken in account, like the connection between metabolic and electromyographic variables proposed in the present study.

The aim of this study was to verify correlations between metabolic (oxygen consumption and cost of transport) and electromyographic (coactivation and electromyographic cost) variables, as well as to obtain the regression equation over a range of walking speeds in CHF and HT patients and healthy controls. A strong positive correlation was hypothesized between cost of transport and electromyographic cost, and between oxygen consumption and the main muscle coactivations in all of the experimental and control groups.

\section{Methods}

\section{Participants}

In the sample calculation, a $20 \%$ difference was considered between the groups of cases (CHF) and the control group, with a statistical power of $80 \%$ and a significance level of $0.05 .{ }^{15}$ The statistical program used was Winpepi, based on data from the study by Figueiredo et al. ${ }^{15}$ and the effect of the sample size was 0.67. For the HT group, all transplant recipients (in the hospital) who were able to walk on the treadmill were invited to participate in the study. The sample was consisted of two experimental groups (CHF and HT patients) and a control group. The first group $(n=12)$ included patients with a previous history of stable symptomatic CHF due to left ventricular systolic dysfunction (left ventricular ejection $<45 \%$ ). Patients with angina, uncontrolled hypertension, renal or pulmonary disease, recent myocardial infarction (previous 3 months), decompensated heart failure, neuromuscular disease, and smokers were not included. The second group $(n=5)$ included all the HT patients of the hospital where the study was carried out. The control group ( $n=12$ ) included individuals matched for age and sex, with a normal medical history and physical examination, as well as with normal resting and exercise ECG. The Institutional Ethics Committee approved the protocol (number 00788512.9.0000.5327), in accordance to the Helsinki Declaration, and all individuals signed an informed consent form (complying with resolution 466/2012).

\section{Study Design}

This was a cross-sectional study, following the Strengthening the Reporting of Observational studies in Epidemiology (STROBE) checklist. ${ }^{16}$ Each participant performed an incremental cardiopulmonary exercise test, whose details are given in Bona et al. (2017), ${ }^{14}$ and, at least $48 \mathrm{~h}$ after, to a walking cost test. 


\section{Cost of transport protocol}

Each experimental session started with 5 minutes of resting $\mathrm{VO}_{2}$ assessment $(\mathrm{m} 102 /(\mathrm{kgmin}))$ in orthostasis. The Self-Selected Walking Speed (SSWS), a comfortable speed that could be sustained for a prolonged period, of each subject was determined according to Figueiredo et al. (2013) $)^{15}$ and Bona et al. ${ }^{17}$ Data acquisitions on treadmill were performed at five different speeds (SSWS; $+/-20 \%$; $+/-40 \%)$, whose sequence was randomized. Each trial lasted 5 minutes in order to reach a steady state for $V_{2}$. Between each speed, the subjects were allowed to rest until the oxygen consumption levels were close to the values of $\mathrm{VO}_{2}$ in orthostasis . Respiratory gas was analyzed breath by breath with a validated system (system (Metalyzer 3B, CPX System; Cortex, Leipzig, Germany), and the cost of transport (C) was estimated from the data collected during the last 2 minutes, by dividing the measured net $\mathrm{VO}_{2}$ (total-resting), adjusted for the respiratory quotient, by the progression speed.

\section{Walking EMG protocol}

Electromyographic signals were collected for 3-5 minutes during each trial, by four miotools 400 (Miotec Biomedical Equipments Ltd, Porto Alegre, Brazil), with a sampling rate of 2,000 $\mathrm{Hz}$ per channel. EMG records were synchronized with digital videos, collected with two CASIO cameras (Exilim FH25; $120 \mathrm{fps}$ ). All procedures recommended by ${ }^{18}$ were strictly observed. Fourteen muscles were monitored on the right side of the body: tibialis anterior (TA), gastrocnemius medialis $(\mathrm{MG})$, vastus lateralis (VL), rectus femoris (RF), biceps femoris (long head, BF), gluteus medius (GM), deltoideus anterior (DA), external oblique (OE), internal oblique (OI), latissimus dorsi (LD), iliocostalis (IC), erector spinae (ES) recorded at $\mathrm{T} 1, \mathrm{~T} 9$, rectus abdominis superior portion (RAS), and trapezius ascending portion (TRAP). Recommended position for electrodes according to SENIAM 2008, except for ES, RAS, ${ }^{19}$ and $\mathrm{OE}^{20}$ were used.

The electromyographic data were analyzed with a custom-written MATLAB program (version 7.1; MathWorks, Inc.), as described in Bona et al. (2016). ${ }^{14}$

\section{Walking EMG analysis}

The EMG data were bandpass filtered with a zero-lag, third-order Butterworth filter with cut-off frequencies at 10 and $500 \mathrm{~Hz}$, and the integral of the rectified EMG (iEMG) signal was determined for each muscle at each phases, with time interpolated over base with 200 points. ${ }^{21}$
The EMG signal was normalized by the peak value of the EMG data. Step phase onsets and offsets were defined using the video recording cameras and three reflective markers (on the fifth metatarsal, calcaneus, and greater trochanter). Sampling of EMG and video recording data were synchronized. For EMG cost, the step was divided by onset, which happened when the heel stuck and offset happened in mid-stance (first stance phase - eccentric contraction), onset happened at mid-stance and offset happened at forefoot lift-off (second stance phase concentric contraction). The balance phase was defined as the interval between the forefoot lift off the ground and the following heel-strike (isometric contraction). ${ }^{22}$ The mean EMG signal was subtracted by the iEMG activity in orthostasis. iEMG signal were converted into count units (100 counts correspond to $\left.1 \mathrm{mV} \cdot \mathrm{s}^{-1}\right) .{ }^{23}$ EMG eccentric $\left(\mathrm{EMG}_{\text {neg }}\right), \mathrm{EMG}$ concentric $\left(\mathrm{EMG}_{\mathrm{pos}}\right)$, and $\mathrm{EMG}$ isometric $\left(\mathrm{EMG}_{\text {iso }}\right)$ were obtained in the counts. The metabolic equivalent was obtained through a linear regression equation of previous experimental study, ${ }^{23}$ as follows:

$$
\begin{array}{cc}
\mathrm{Vo}_{\text {2neg }}(\mathrm{ml})=0.0015 . \mathrm{EMG}_{\text {neg }}+0.3353 & \text { Equation 1 } \\
\mathrm{Vo}_{\text {2pos }}(\mathrm{ml})=0.0042 . \mathrm{EMG}_{\text {pos }}+0.1493 & \text { Equation 2 } \\
\mathrm{Vo}_{\text {2iso }}(\mathrm{ml})=0.0042 \cdot \mathrm{EMG}_{\text {iso }}+0.1394 & \text { Equation 3 }
\end{array}
$$

$\mathrm{VO}_{2 \text { neg }}$ is the oxygen consumption for negative mechanical work, $\mathrm{VO}_{2 \text { pos }}$ is the oxygen consumption for positive mechanical work, and $\mathrm{VO}_{2 \text { iso }}$ is the oxygen consumption for isometric contraction.

Finally, the sum of metabolic equivalent was converted into Joules using an energetic equivalent. This was obtained adjusting the respiratory quotient. ${ }^{24}$ Subsequently, this value was divided by step length to determine the walking EMG cost in joules per meter (equation 4$)$.

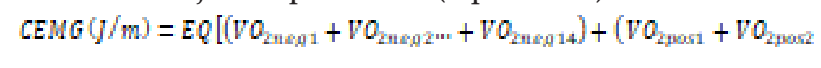

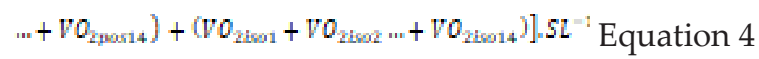

EQ is the energetic equivalent, SL is the stride length.

The percentage of coactivation was defined by the onset of the stance phase, when the calcaneus touched the ground, and the offset, when the forefoot lifted off the ground; the balance phase was defined as described above. ${ }^{23}$ Coactivation was analyzed for five pairs of muscles, DA-LD, IC-OI, ES-RAS, TA-GM, and RF-BF, by the quotient between antagonistic iEMG and agonistic iEMG (as follows) at each phase described above. ${ }^{25}$

$$
\text { Coactivation }=\frac{i \text { EMGantagonist }}{i \text { EMGagonist }} X 100 \text { Equation } 5
$$




\section{Data analysis}

Data analysis was based upon a linear model. The relation between CEMG and $\mathrm{C}$ was analysed through a standard major axis (SMA) linear regression model, following the recommendations of Sokal and Rohlf. ${ }^{26}$ The four assumptions associated with a linear regression model were verified as follows:

1) Normality: by means of the Shapiro-Wilk test.

2) Homoscedasticity: by means of the Levene test.

3) Linearity: through the analysis of the Akaike Information Criterion (AIC) for linear and non-linear models.

4) Independency: by the Durbin-Watson statistics, testing the autocorrelation of the residuals.

All the tests gave no significant results at alpha $=0.05$, and all the best likelihood of AIC were in favor of linear models. Therefore, SMA linear regression equations were calculated using the mean values of CEMG and $\mathrm{C}$. The regression equation ( $y=a+b x$; where" $b^{\prime \prime}$ is the slope) was obtained by estimating CEMG $\left(\mathrm{J}^{\mathrm{kg}}{ }^{-1} \mathrm{~m}^{-1}\right)$ from C for the general population and for each group of this study. Due to the properties of SMA linear regression, where $x$ and $y$ are interchangeable, the equations can be adjusted to estimate $C$ from CEMG as well ${ }^{26}$ The results of regression equations are dependents of the $\mathrm{C}$ speed used during walking.

Pearson's correlation coefficient ( $r$ ) was used to assess the linear relationship between oxygen consumption and coactivation.

One-way ANOVA was carried out to test for differences between groups for each anthropometric, cardiopulmonary, and cost measures. Normality and homoscedasticity of all variables was verified as specified above, and the sphericity by the Mauchly test. When appropriate, multiple comparisons were made with Bonferroni correction. Continuous variables are presented in mean and standard deviation, categorical (gender and medicine) data were described in absolute frequencies.

Analyses have been performed using the SPSS statistical package (IBM, USA), version 13.0, and $R$, version 3.6.3, with alpha $=0.05$.

\section{Results}

\section{Participants' characteristics}

As shown in Table 1 , the age and gender of CHF patients and controls were well matched. All patients with HT in
Hospital de Clínicas de Porto Alegre (Clinical Hospital of Porto Alegre, Brasil) participated in this study. HT Patients presented a higher mean weight than did CHF patients $(\mathrm{P}=0.006)$, and a higher height than the controls and $\mathrm{CHF}$ patients ( $\mathrm{P}=0.003$ and 0.001 , respectively). CHF Patients showed a moderate reduction in the left ventricular systolic function and a mild impairment in functional capacity. SSWS of CHF and HT patients was lower than that of the controls ( $\mathrm{P}=0.001 / 0.001)$, while $\mathrm{C}$ was higher in the same groups $(\mathrm{P}=0.001 / 0.001)$. All $\mathrm{CHF}$ patients were studied under currently recommended medical therapy. $V O_{2 \text { peak }}$ was higher in the control group $(\mathrm{P}=0.001)$. The VE/ $V C O_{2 s l o p e}$ was significantly higher in CHF and HT patients $(\mathrm{P}=0.049 / 0.027)$. Peak heart rate and $\%$ of predicted and peak heart rate were higher in the control group (0.001/0.001). The $V E / V C O_{2}$ was higher in the HT group. As expected, controls presented the lowest $\mathrm{C}$ and CEMG at the SSWS ( $\mathrm{P}=<0.001 / 0.001$ and $<0.001 /<0.001)$.

Data are mean \pm SD or number of subjects. $\mathrm{CHF}$, chronic heart failure; HT, heart transplant; $\mathrm{f} / \mathrm{m}$, female/male; ACE-I, angiotensin-converting enzyme inhibitor; ARA, angiotensin receptor antagonist; $V_{2}$ peak, peak oxygen uptake; $V E\left(V C \mathrm{O}_{2}\right)$ slope, minute ventilation to carbon dioxide production slope; OWS Froude, OWS calculated by Froude number; 220 - age, 220 minus the subject's age; SSWS self-selected walking speed; C cost of transport; CEMG electromyographic cost. Symbols identify statistical significance in the Bonferroni test: ${ }^{* *}$ differences between HT and others; *** differences between controls and others; **** difference between HT and CHF. * identifies statistical significance; + differences between HT and controls; $\ddagger$ differences between $\mathrm{HT}$ and CHF; § differences between controls and CHF; Data source: B ona et al. 2017. ${ }^{14}$

\section{Walking EMG: correlations and regression}

The values of Pearson's correlation coefficients between oxygen consumption and muscle coactivation, during stance and balance phases at five walking speeds are shown in Table 2. Coactivation were determined between five couples of muscles (deltoideus anterior - latissimus dorsi; iliocostalis - internal oblique; erector spinae - rectus abdominis; tibialis anterior - gastrocnemius; rectus femoris - biceps femoris) . Values of Pearson's correlation coefficient (r) were around 0.87 and 0.98 , classified as strong correlation, ${ }^{27}$ for tree groups (controls, $\mathrm{CHF}$, and HT patients), and was significant according the table. For information about CA comparisons between groups (see supplementary document and Bona et al., 2017). 
Table 1 - Characteristics of controls and CHF and HT patients

\begin{tabular}{|c|c|c|c|c|}
\hline & Controls & CHF & HT & p-value \\
\hline & $(n=12)$ & $(n=12)$ & $(n=5)$ & \\
\hline Age (yr) & $57 \pm 11$ & $59 \pm 10$ & $57 \pm 6$ & \\
\hline Weight (kg) & $70.3 \pm 14$ & $66 \pm 12$ & $82 \pm 7^{* * * *}$ & 0.006 \\
\hline Gender (f/m) & $4 / 8$ & $4 / 8$ & $1 / 4$ & \\
\hline Height $(\mathrm{cm})$ & $166 \pm 11$ & $160 \pm 12$ & $180 \pm 10^{* *}$ & $0.003+/ 0.001 \ddagger$ \\
\hline BMI $\left(\mathrm{kg} / \mathrm{m}^{2}\right)$ & $25.51 \pm 6.1$ & $25.78 \pm 5.9$ & $25.30 \pm 7.2$ & 0.1 \\
\hline Time after HT (yr) & - & - & $1.7 \pm 1$ & \\
\hline Left ventricular ejection fraction (\%) & - & $33 \pm 3$ & $70 \pm 9$ & \\
\hline Beta-blockers & - & 12 & 5 & \\
\hline Diuretics & - & 10 & 3 & \\
\hline ACE-I/ARA & - & 12 & 5 & \\
\hline Digoxin & - & 7 & - & \\
\hline Anticoagulants & - & 8 & 5 & \\
\hline Immunosuppressor & - & & 5 & \\
\hline $\mathrm{VO}_{2}$ peak $\left(\mathrm{mL} \cdot \mathrm{kg}^{-1} \mathrm{~min}^{-1}\right)$ & $32.1 \pm 6.3^{*}$ & $23.9 \pm 5.1$ & $19.3 \pm 2.9$ & 0.001 \\
\hline $\mathrm{VE}\left(\mathrm{VCO}_{2}\right)$ slope & $28 \pm 2 * * *$ & $32 \pm 1$ & $35 \pm 4$ & $0.049 \S / 0.027 / /$ \\
\hline Peak expiratory exchange ratio & $1.22 \pm 0.07$ & $1.15 \pm 0.05$ & $1.27 \pm 0.03$ & 0.073 \\
\hline Peak heart rate, $\%$ of predicted (220-age) & $110 \pm 3^{* * *}$ & $87 \pm 2$ & $76 \pm 2$ & $0.001 \S / 0.001 / /$ \\
\hline Peak heart rate (bpm) & $179 \pm 3^{* * *}$ & $139 \pm 2$ & $124 \pm 4$ & $0.001 \S / 0.001 / /$ \\
\hline $\begin{array}{l}\text { Peak } \\
\mathrm{VE}\left(\mathrm{VC}_{2}\right)\end{array}$ & $31.11 \pm 4.1$ & $32.83 \pm 3.9$ & $38.86 \pm 3.2^{* *}$ & $0.022+/ 0.028 \ddagger$ \\
\hline SSWS $\left(\mathrm{m} \cdot \mathrm{s}^{-1}\right)$ on treadmill & $1.04 \pm 0.4^{* * *}$ & $0.75 \pm 0.3$ & $0.85 \pm 0.3$ & $0.001 \S / 0.011 / /$ \\
\hline C at the SSWS (J.kg.m-1) & $1.93 \pm 0,71^{* * *}$ & $3.04 \pm 0.8$ & $3.01 \pm 0.31$ & $<0.001 \S / 0.001 / /$ \\
\hline CEMG at the SSWS $\left(\mathrm{J}^{-\mathrm{m}^{-1}}\right)$ & $2.77 \pm 0.9^{* * *}$ & $3.52 \pm 1.2$ & $3.16 \pm 1.3$ & $<0.001 \S /<0.001 / /$ \\
\hline $\mathrm{VO}_{2}$ at the SSWS $\left(\mathrm{mL} \cdot \mathrm{kg}^{-1} \mathrm{~min}^{-1}\right)$ & $11.77 \pm 0.6$ & $10.59 \pm 0.9$ & $11.53 \pm 0.9$ & 0.26 \\
\hline \multicolumn{5}{|c|}{$\begin{array}{l}\text { Data are mean } \pm \text { SD or number of subjects. CHF, chronic heart failure; HT, heart transplant; flm, female/male; ACE-I, angiotensin-converting } \\
\text { enzyme inhibitor; ARA, angiotensin receptor antagonist; peak, peak oxygen uptake; () slope, minute ventilation to carbon dioxide production slope; } \\
\text { OWS Froude, OWS calculated by Froude number; } 220 \text { - age, } 220 \text { minus the subject's age; SSWS self-selected walking speed; C cost of transport; } \\
\text { CEMG electromyographic cost. Symbols identify statistical significance in the Bonferroni test: }{ }^{* *} \text { differences between HT and others; }{ }^{* * *} \text { differences } \\
\text { between controls and others; }{ }^{* * * *} \text { difference between HT and CHF. }{ }^{*} \text { identify statistical significance; }+ \text { differences between HT and controls; } \neq \text { differences } \\
\text { between HT and CHF; } \S \text { differences between controls and CHF; Data source: Bona et al. } 2017 . .^{14}\end{array}$} \\
\hline
\end{tabular}

Correlation coefficient between the oxygen consumption and coactivation of DA-LD (deltoideus anterior - latissimus dorsi), IC-OI (iliocostalis internal oblique), ES-RAS (erector spinae - rectus abdominis superior portion), TA-GM (tibialis anterior gastrocnemius medialis), RF-BF (rectus femoris - biceps femoris long head) during stance and balance phases at five walking speeds.
Differences between groups for CA (see supplementary document) were discussed in Bona et al., 2017. ${ }^{14}$

The regression equations for estimating CEMG $\left(\mathrm{J} \cdot \mathrm{kg}^{-1} \cdot \mathrm{m}^{-1}\right)$ from $\mathrm{C}$, in a range of speed around the SSWS, were calculated in four models:

i) general equation: $\mathrm{CEMG}=0.547+0.921(\mathrm{C})$;

ii) controls: $\mathrm{CEMG}=-0.091+1.209(\mathrm{C})$; 
Table 2 - shows the Pearson correlation between oxygen consumption $\left(\mathrm{VO}_{2}\right)$ and coactivation $(\mathrm{CA})$ - stance and balance phases

\begin{tabular}{|c|c|c|c|c|c|c|}
\hline \multirow[b]{2}{*}{ Correlation } & \multicolumn{2}{|c|}{ Controls } & \multicolumn{2}{|r|}{$\mathrm{CHF}$} & \multicolumn{2}{|c|}{ HT } \\
\hline & $r$ & $\mathrm{p}$ & & $\mathrm{p}$ & & $\mathrm{p}$ \\
\hline \multicolumn{7}{|c|}{$\mathrm{VO}_{2}$ and CA Stance Phase } \\
\hline DA-GD & 0.91 & 0.001 & 0.84 & 0.033 & 0.94 & 0.024 \\
\hline IL-OI & 0.95 & 0.038 & 0.70 & 0.04 & 0.78 & 0.03 \\
\hline ES-RAS & 0.96 & 0.032 & 0.87 & 0.026 & 0.96 & 0.001 \\
\hline TA-GM & 0.95 & 0.001 & 0.90 & 0.001 & 0.98 & 0.001 \\
\hline RF-BF & 0.92 & 0.001 & 0.89 & 0.034 & 0.94 & 0.002 \\
\hline \multicolumn{7}{|c|}{$\mathrm{VO}_{2}$ and CA Balance Phase } \\
\hline DA-GD & 0.92 & 0.03 & 0.8 & 0.03 & 0.98 & 0.001 \\
\hline IL-OI & 0.98 & 0.001 & 0.82 & 0.032 & 0.92 & 0.001 \\
\hline ES-RAS & 0.90 & 0.002 & 0.86 & 0.041 & 0.88 & 0.01 \\
\hline TA-GM & 0.87 & 0.035 & 0.90 & 0.021 & 0.92 & 0.026 \\
\hline RF-BF & 0.94 & 0.001 & 0.93 & 0.04 & 0.89 & 0.039 \\
\hline \multicolumn{7}{|c|}{$\begin{array}{l}\text { Correlation coefficient between the oxygen consumption and coactivation of DA-LD (deltoideus anterior - latissimus dorsi), IC-OI (iliocostalis - internal } \\
\text { oblique), ES-RAS (erector spinae - rectus abdominis superior portion), TA-GM (tibialis anterior - gastrocnemius medialis), RF-BF (rectus femoris - biceps } \\
\text { femoris long head) during stance and balance phases at five walking speeds. CHF-chronic heart failure; HT - heart transplant. } r \text { is Pearson's correlation } \\
\text { coefficient. P significant correlation values. }\end{array}$} \\
\hline
\end{tabular}

iii) $\mathrm{CHF}$ : $\mathrm{CEMG}=0.630+0.871(\mathrm{C})$;

iv) HT: $\mathrm{CEMG}=0.221+1.047(\mathrm{C})$;

where CEMG is the electromyographic cost and $\mathrm{C}$ is the cost of transport.

Linear regression graphs are presented in figure 1. All values for $\mathrm{R}^{2}$ were between 0.88 and 0.99 , which validated the model. For controls, the linear model explains $99 \%$ for the $\mathrm{C}$ values, $92 \%$ for $\mathrm{CHF}$, and $88 \%$ for $\mathrm{HT}$.

\section{Discussion}

Better understanding of complex interactions involved in measuring mechanical work and efficiency might best be obtained using a multidisciplinary approach. ${ }^{28}$ The present study proposed an evaluation of the relationship between electromyographic and physiological variables, using specific (coactivation percentage) and global ( $\mathrm{C}$ and CEMG) measures. The global measures reflect the sum of more localized contributions throughout the body.

The linear regression analyses showed a strong relationship between CEMG and C in the three analyzed populations. However, the metabolic cost appears slightly underestimated in the controls, but the CEMG seems to be a bit overestimated, with HT set in between, with the slope at nearly 1 (Figure 1). As shown, it is possible to estimate the $C$ towards the CEMG in the three analyzed populations. CEMG could replace $C$ in some clinical situations: when a gas analyzer is not available, when using a mask is not recommended (like in cerebral palsy), or when the population present some alteration in respiratory parameters (like in a respiratory disease), which could to change the real values.

A strong and significant correlation was found in all the analyzed groups, between the CEMG and the metabolic $\mathrm{C}$, and between oxygen consumption and the main muscle coactivation. According to Bona et al. ${ }^{17}$ the mechanical work was higher, while the mechanical efficiency was lower in CHF and HT, when compared with the controls. Those differences were partially due to differences of SSWS. However, muscle activation and CEMG also play a secondary role. ${ }^{14}$ According to Williams (1985), ${ }^{28}$ muscular efficiencies are typically between $20-35 \%$ for walking. Mechanical efficiency during walking was near $25 \%$ at SSWS. For CHF and 

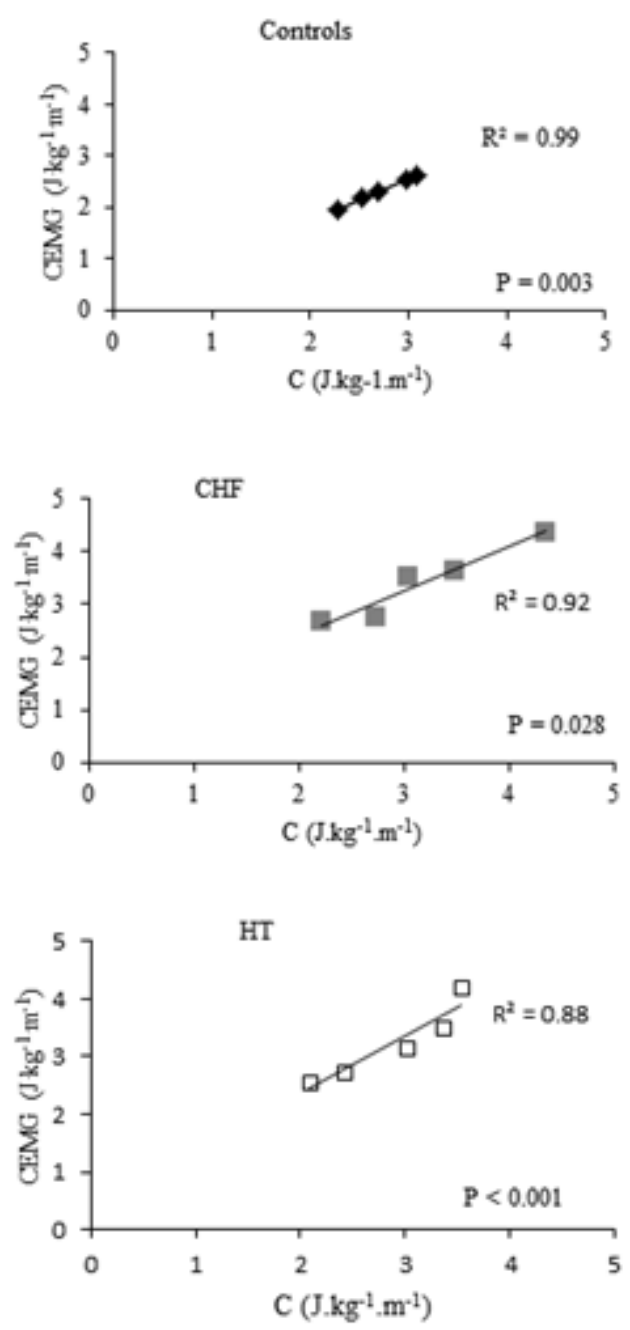

Figure 1-C is the cost of transport, CEMG is the electromyoghraphic cost, CHF is chronic heart failure, HT is heart transplant. R2 is the determination coefficient. Significant correlation $(p<0.05)$.

HT, the mechanical efficiency during walking achieve values near $20 \%$ at the same speed of the control group. ${ }^{17}$

The EMG technique presents some limitations, much like other physiological evaluations. EMG recordings provide a local quantitative measure of total muscle fiber activity, and changes in total rate of oxygen uptake result primarily from changes in the oxygen uptake of the general working of muscles. ${ }^{23} \mathrm{~A}$ strong relationship was reported between EMG data and mechanical work. ${ }^{29}$ Decrease in the recovery index and increase in the external mechanical work in CHF and HT patients is determined by a reduction in the pendulum-like energy interchange due to an altered trajectory of the COMB. ${ }^{17}$ The authors suggest that the reported differences in external mechanical work and recovery could determine an increased muscle activity to sustain locomotion. All these data confirm the relationship between EMG data and biomechanical parameters during walking.

Analysis of the energy recovery showed that, according to literature, the exchange between kinetic energy and potential energy was limited, especially at slow speeds, while it reached maximum values at the higher walking speeds. ${ }^{30-32}$ By optimizing the system conversion, less metabolic energy is needed to keep walking. The SSWS adopted by CHF and HT patients directly determines the efficiency of the pendulum mechanism (recovery). Reducing their speed, patients increase the stability of walking, but increase the variability of movements as well, 33,34 a factor that could affect the muscular activity and, therefore, the C. Improvements in walking speed, 
leading to the optimal walking speed, would increase the vertical displacement of the $\mathrm{COMB}$, providing larger oscillations of kinetic energy and potential energy of the COMB and, generally, an improvement of the efficiency of the pendulum mechanism of walking.

The rate of oxygen consumption was significantly correlated to leg, trunk, and arm muscle activation in all groups, suggesting that these are key factors affecting exercise performance. This finding suggests that dynamic muscle coactivation was an important factor specific to CHF and HT as a quality of movement index. The authors estimated coactivations in both stance and balance phases of walking, and at specific phases and requests of movement. These data support the idea that peripheral muscle limitations play an important role in people with $\mathrm{CHF}$ and $\mathrm{HT}$, confirming and extending prior studies. ${ }^{14,17}$ The same behavior has been shown for a number of other muscles and for a variety of different types of dynamic contractions. ${ }^{29}$

There is compelling evidence that skeletal muscle dysfunctions play an important role in exercise intolerance in $\mathrm{CHF}$. The problem involves the large muscles of locomotion, small muscles of the arms, and even respiratory muscles. These changes lead to increased muscle fatiguability, decreased oxidative metabolism, increased oxidative stress, and ineffective high energy phosphate, most likely resulting in an early accumulation of lactate during walking.

The beneficial effect of exercise training on reducing skeletal muscle alterations in these patients demonstrates that this process is reversible. ${ }^{35}$ The changes lead to an improvement in peak $\mathrm{VO}_{2}$ and lactate threshold, and delayed onset of anaerobic metabolism. Exercise training also has anti-inflammatory effects, and, as described above, can increase local expression of the anabolic peptide insulin-like growth factor 1. Additionally, reduced sympathetic hyperactivation and improved endothelial dysfunction with exercise training contribute to improved muscle blood flow and clinical performance. ${ }^{35}$

One recent study ${ }^{36}$ found increased coactivation in people with multiple sclerosis when compared to healthy subjects walking at similar speeds. This fact appeared not to be due to different gait velocities for this population; by contrast, it was likely an adopted strategy, suggesting that this mechanism may be part of a characteristic implemented pattern that may explain differences in gait ability. For $\mathrm{CHF}$ and HT patients, this increase in percentage of coactivation may well contribute to higher C and CEMG, ${ }^{14}$ lower recovery, and mechanical efficiency at SSWS. ${ }^{17}$
Another study ${ }^{37}$ analyzed the activity of agonist and antagonist muscles during gait in patients with multiple sclerosis, demonstrating that abnormal lower limb muscle coactivation occurs during the stance phase of gait. This increased lower limb muscle coactivation during gait is an adaptive strategy that tends to compensate for muscle weakness. The relationship between muscle coactivation during gait and disability, postural stability, joint stiffness, and gait performance are regulated by variations in the forces produced. This is important, since an inappropriate coactivation (excessive and/ or prolonged) reduces gait performance by reducing gait speed and increasing metabolic cost. The results of the present study increase understanding of the function of coactivation in cardiac patients.

Research in medicine sports for decades has demonstrated that the skeletal muscle function improved through exercise training, which reduces the debilitating symptoms of chronic heart failure through its effects on the cardiovascular and musculoskeletal systems. Analysis of muscle activation can provide important clinical information and the ability of patients to produce a desired functional outcome. ${ }^{36}$ In these way, mortality and hospital admission are significantly reduced after exercise training in patients with $\mathrm{CHF}$, and this benefit was not restricted to any particular subgroup of patients.

The present study proposes that for CHF and HT patients who have SSWS lower than OWS, rehabilitation programs should focus on improving the speed of exercise, such as dynamic muscle contraction (and coactivation) for a better efficiency and economy of movement. Thus, further studies are necessary to evaluate other populations, like chronic obstructive pulmonary disease, multiple sclerosis, or cerebral palsy with these methods.

It is important to consider some limitations of the present study. The SSWS on the ground, for all groups, was greater than SSWS on the treadmill. This, as described by Figueiredo et al. (2013), ${ }^{15}$ may have affected the results (see Table 1). SSWS are expected to be lower on the treadmill than on the ground, hence the control group's lower SSWS. The assessment of the SSWS is an important task in an attempt to define the characteristics of walking and the physical tolerance of each group. Our CHF patients showed only a slight decrease in function capacities and, for this reason, our outcomes cannot be extended to patients with more severe CHF. However, with the current clinical therapies, more and more patients of CHF clinics have a profile equivalent to that of our group. 


\section{Conclusion}

The aim of this study was to verify the correlation between oxygen consumption and coactivation, as well as the correlation between C and CEMG in CHF and HT patients and healthy controls. Accordingly, the results highlighted complementary strategies to reduce the $C$ caused by muscle economy denominated CEMG, in addition to the inverted pendulum mechanism. More specifcially, muscle coactivation seemed to play a role in the increase of oxygen consumption throughout postural stability, stance, and balance phases for the entire gait cycle to determine total body energy consumption. Identification of inappropriate activation and how this may lead to specific locomotor deficits could lead to more effective training interventions.

\section{Clinical Messages}

Strategies to increase muscle economy and stability, reducing muscle contraction, can help the physical condition and locomotion of patients with heart disease.

\section{Acknowledgements}

The authors declared no potential conflicts of interest regarding the research, authorship, and/or publication of this article.

\section{Potential Conflict of Interest}

No potential conflict of interest relevant to this article was reported.

\section{References}

1. Margaria R. Biomechanics and energetics of muscular exercise. Oxford (UK): Clarendon Press. 1976.

2. Margaria R, Cerretelli P, Aghemo P, Sassi G. Energy cost of running. J Appl Physiol. 1963; 18(2):367-70.

3. Cotes JE, Meade F. The energy expenditure and mechanical energy demand in walking. Ergonomics. 1960; 3(2):97-120.

4. Cavagna GA, Heglund NC, Taylor CR. Mechanical work in terrestrial locomotion: two basic mechanisms for minimizing energy expenditure. Am J Physiol Regul Integr Comp Physiol. 1977; 233(5): R243-R261.

5. Carrier DR, Anders C, Schilling N. The musculoskeletal system of humans is not tuned to maximize the economy of locomotion. Proc Natl Acad Sci U S A. 2011; 108 (46): 18631-6.

6. Cunningham CB, Schilling N, Anders C, Carrier DR. The influence of foot posture on the cost of transport in humans. J Exp Biol. 2010; 213 (5):790-7.

7. Bramble DM, Lieberman DE. Endurance running and the evolution of Homo. Nature. 2004; 432(7015):345-52.

\section{Sources of Funding}

This study was partially funded by Fundo de Incentivo à Pesquisa e Eventos (FIPE).

\section{Study Association}

This article is part of the thesis of Doctoral submitted by Renata Luísa Bona, from Programa de Pós-graduaçao em Ciências da Saúde: Cardiologia e Ciências Cardiovasculares, Hospital de Clínicas de Porto Alegre, Universidade Federal do Rio Grande do Sul.

\section{Ethics approval and consent to participate}

This study was approved by the Ethics Committee of the Hospital de Clínicas de Porto Alegre / Plataforma Brasil under the protocol number 00788512.9.0000.5327. All the procedures in this study were in accordance with the 1975 Helsinki Declaration, updated in 2013. Informed consent was obtained from all participants included in the study.

\section{Author contributions}

Conception and design of the research: Bona RL, Bonezi A, Castro FAS, Clausell N. Acquisition of data: Bona RL, Bonezi A. Analysis and interpretation of the data: Bona RL, Bonezi A, Biancardi CM, Castro FAS, Clausell N. Statistical analysis: Bona RL, Biancardi CM. Obtaining financing: Bona RL, Clausell N. Writing of the manuscript: Bona RL, Bonezi A, Biancardi CM, Castro FAS, Clausell N. Critical revision of the manuscript for intellectual content: Bona RL, Bonezi A, Biancardi CM, Castro FAS, Clausell N.

8. Wilson JR, Martin JL, Schwartz D, Ferraro N. Exercise intolerance in patients with chronic heart failure: role of impaired nutritive flow to skeletal muscle. Circulation. 1984; 69 (6): 1079-87.

9. Sullivan MJ, Knight JD, Higginbotham MB, Cobb FR. Relation between central and peripheral hemodynamics during exercise in patients with chronic heart failure. Circulation. 1989;80 (4):769-81.

10. Mancini DM, LeJentel TH, Factor S, Sonnenblick E. Central and peripheral components of cardiac failure. Am J Med. 1986; 80 (2):2-13.

11. Wilson JR, Mancini DM, Dunkman WB. Exertional fatigue to skeletal muscle dysfunction in patients with heart failure. Circulation. 1993;87 (2):470-5.

12. Wilson JR, Mancini DM, Simson M. Detection of skeletal muscle fatigue in patients with heart failure using electromyography. Am J Cardiol. 1992; 70 (4): 488-93.

13. Schulze PC, Linkea A, Schoene N, Winklera SM, Adamsa V, Conradib S, Bussec M, Schulera G, Hambrechta R. Functional and morphological skeletal muscle abnormalities correlate with reduced electromyographic activity in chronic heart failure. Eur J Prev Cardiol. 2004; 11 (2): 155-61. 
14. Bona RL, Bonezi A, Silva PF, Biancardi CM, Castro FADS, Clausel NO. Electromyography and economy of walking in chronic heart failure and heart transplant patients. Eur J Prev Cardiol. 2017; 24 (5): 544-51.

15. Figueiredo PF, Ribeiro PB, Bona RL, Tartaruga LA, Ribeiro JP. Ventilatory determinants of self-selected walking speed in chronic heart failure. Med Sci Sports Exerc. 2013; 45 (13): 415-9.

16. Von Elm E, Altman DG, Egger M, Pocock SJ, Gøtzsche PC, Vandenbroucke JP. The Strengthening the Reporting of Observational Studies in Epidemiology (STROBE) statement: guidelines for reporting observational studies. Ann Intern Med. 2008; 147(8), 573-7.

17. Bona RL, Bonezi A, Silva PF, Biancardi CM, de Souza Castro FA, Clausel NO. Effect of walking speed in heart failure patients and heart transplant patients. Clin Biomech. 2017; 42: 85-91.

18. Hermens HJ, Freriks B, Disselhorst-Klug C, Raul G. Development of recommendations for SEMG sensors and sensor placement procedures. J Electromyogr Kinesiol. 2000; 10 (5): 361-74.

19. Winter DA. The Biomechanics and Motor Control of Human Gait: Normal, Elderly and Pathological. 2nd ed. Ontario: Waterloo Biomechanics; 1991.

20. Escamilla RF, Babb E, Dewitt R, Jew P, Kelleher P, Burnham T, et al. Electromyographic analysis of traditional and nontraditional abdominal exercises: implications for rehabilitation and training. Phys Ther. 2006; 86 (5): 656-71.

21. Cappellini G, Ivanenko YP, Poppele RE, Lacquaniti F. Motor patterns in human walking and running. J Neurophysiol. 2006; 95 (6): 3426-37.

22. Sinkjòr T, Andersen JB, Ladouceur M, Christensen LO, Nielsen JB. Major role for sensory feedback in soleus EMG activity in the stance phase of walking in man. J Physiol. 2000; 523 (3): 817-27.

23. Bigland-Ritchie B, Woods JJ. Integrated electromyogram and oxygen uptake during positive and negative work. J physiol. 1976; 260 (2): 267-77.

24. Cooke CB. Metabolic rate and energy balance. In: Eston R and Reilly T (eds) Kinanthropometry and Exercise Physiology Laboratory Manual Tests, Procedures and Data, Abingdon: Routledge; 2009. pp.147-173.
25. Osterning LR, Hamill $\mathrm{J}$ and Lander JE. Co-activation of sprinter and distance runner muscles in isokinetic exercise. Med Sci Sports Exerc. $1986 ; 18$ (4): 431-5.

26. Sokal R. R.; Rohlf F. J. Biometry: The principles and practice of statistics in biological research. San Francisco (CA): W. H. Freeman; 1995.

27. Field A. Discussing Statistics using SPSS. 3rd ed. London: Sage Publications; 2009.

28. Williams KR. The relationship between mechanical and physiological energy estimates. Med Sci Sports Exerc. 1985; 17 (3):317-25.

29. Bouisset $S, F$ Goubel. Integrated electromyographical activity and muscle work. J Appl Physiol. 1973; 35 (5): 695-702.

30. Cavagna GA, Thys H, Zamboni A. The sources of external work in level walking and running. J Physiol. 1976; 262 (3): 639-57.

31. Willems PA, Cavagna GA, Heglund NC. External, internal and total work in human locomotion. J Exp Biol. 1995; 198 (2): 379-93.

32. Pavei G, Biancardi CM, Minetti AE. Skipping vs. running as the bipedal gait of choice in hypogravity. Am J Physiol Heart Circ Physiol. 2015; 119 (1): 93-100.

33. England SA, Granata KP. The influence of gait speed on local dynamic stability of walking. Gait Posture. 2007; 25: 172-8.

34. Kang HG, Dingwell JB. Effects of walking speed, strength and range of motion on gait stability in healthy older adults. J biomec. 2008; 41 (14):2899-905

35. Piepolic MF. Exercise training in chronic heart failure: mechanisms and therapies Netherlands Heart Journal. 2013; 21(2): 85-90.

36. Lencioni T, Jonsdottir J, Cattaneo D, Crippa A, Gervasoni E, Rovaris M, et al. Are modular activations altered in lower limb muscles of persons with multiple sclerosis during walking? Evidence from muscle synergies and biomechanical analysis. Front Hum Neurosci. 2016; 10: 620- limb muscles during gait in patients with multiple sclerosis. PloS One. 2016; 11 (6): 1-13. 33. 